\title{
A Model-driven Approach for the Description of Blockchain Business Networks
}

\author{
Stefan Seebacher ${ }^{1}$ \\ Karlsruhe Institute of Technology \\ stefan.seebacher@kit.edu
}

\author{
Maria Maleshkova ${ }^{1}$ \\ Karlsruhe Institute of Technology \\ maria.maleshkova@kit.edu
}

\begin{abstract}
The concept of blockchain technology has gained significant momentum in practice and research in the past few years, as it provides an effective way for addressing the issues of anonymity and traceability in distributed scenarios with multiple parties, which have to exchange information and want to securely collaborate with each other. However, up-to-date, the impact of the structure and setup of business networks on successfully applying blockchain technology, remains largely unexplored.

We propose a model-driven approach, combining an ontology and a layer model, that is capable of capturing the properties of existing blockchain-driven business networks. The layers are used to facilitate the comprehensive description of such networks. We also introduce the Blockchain Business Network Ontology (BBO), formalizing the concepts and properties for describing the integral parts of a blockchain network. We show the practical applicability of our work by evaluating and applying it to an available blockchain use case.
\end{abstract}

\section{Introduction}

On the one hand, for many researchers and practitioners blockchain technology brings the promise of revolutionizing the way we interact and transact over the Internet (e.g. see [1]), as the technology facilitates the formation of self-sufficient and distributed networks. On the other hand, products and services are more and more developed and offered in a distributed manner (see e.g. [2]). Companies have multiple locations, the involved business partners and customers are located in different cities, and interactions take place in an ubiquitous manner, sometimes without even knowing who the interacting

\footnotetext{
${ }^{1}$ Both authors contributed equally to this work.
}

parties are. Amongst others, due to the advent of the Internet, such business network setups are increasingly characterized by anonymity and are, therefore, in need of a trust-evoking authority or a reliable intermediary. In this context, blockchain technologies provide a solution to the issues of anonymity and traceability in distributed scenarios, with multiple parties that have to exchange information and engage in a collaborative manner. Despite its benefits, the adoption of blockchain technology still remains on a low level, as emerging blockchain solutions face strong competition from mature and well-established systems [3] as well as the dissemination of persuasive use cases is still insufficient [4]. Most importantly, the systematic support for putting blockchain solutions into practice is missing. One significant part of providing this support, is having a foundation for understanding the relationships between the business network setup and the blockchain solution. In particular, certain network setups could be more suitable for blockchain-based solutions, while in other cases blockchains might not be reasonable or even harm the business activities of involved parties. However, up-to-date, this relationship, and the resulting requirements remain largely unexplored. Therefore, a major hurdle for "the design of intra- as well as inter-enterprise information systems and the deployment of modern ICT in implementing these systems" is "the lack of an appropriate, deep understanding of enterprises and enterprise networks" [5].

In this context, we offer a common basis for describing and understanding existing business network setups. In detail, we make the following contributions:

- C1: A layer-based model for capturing the network setup of a particular blockchain use case. Here, we focus on providing the means for describing a use case based on three main levels: 1) business model, 2) network composition, and 3) technical implementation.

- C2: The BBO - Blockchain Business Network Ontology, which provides concepts and properties for describing all integral parts of a blockchain 
network. The BBO serves as a model for specifying and formalizing blockchain networks.

- C3: Assessment of a use case, in order to show the practical applicability and to evaluate our approach.

Based on these contributions, we establish a common and shared model for describing the integral parts of a blockchain network. One advantage is that the resulting description gives a formalization of the current state of the network, which all participating parties can agree on. Furthermore, it can be used as the basis for analyzing the suitability of implementing a blockchain solution and deriving the corresponding requirements. Finally, it may serve as the starting point for tracking changes, determining problems, weaknesses and areas for improvement. Building upon the layer model and BBO, approaches for the automated assessment and deployment of blockchain solutions can be developed, potentially facilitating a wider adoption of blockchain technology.

This paper is structured as follows: Section 2 provides the theoretical foundations of our work, describing the concepts of blockchain technology, ontologies and model-driven solutions. Section 3 gives an overview of related work, while Section 4 describes the applied methodology. Section 5 introduces our layer model and the BBO. In Section 6, we evaluate the developed ontology by applying it to a use case and discussing our findings. We conclude the paper with a summary of our contributions and an outlook on future work in Section 7.

\section{Theoretical Foundations}

In this section, we give a brief introduction to three theoretical areas, which play a crucial role for our work. These are: 1) blockchain technology, 2) ontologies and ontology engineering, and 3) modeldriven architectures. These fundamentals lay out the basis for developing a model and capturing the properties of blockchain business networks.

Blockchain Technology. Blockchain technology was initially developed to enable the creation of the cryptocurrency Bitcoin [6]. The concept of blockchain technology as well as its main notions are given below [7]:

- A blockchain is a distributed and shared database

- It consists of a linked sequence of blocks, holding secured time-stamped transactions

- Once an element is appended to the blockchain, it can not be altered

In essence, a blockchain can be regarded as a distributed ledger technology, which on the one hand provides a platform for participants to interact with each other and on the other hand serves as a transparent, comprehensible and trustworthy repository of data and information (see e.g. [3], [7], [8]). There is a variety of interrelated characteristics, which can be attributed to the technology. A comprehensive overview is given in Seebacher and Schüritz [9]. In this context, the two main elements of blockchain technology are its trust evoking and decentralized nature. On the one hand, trust is facilitated through a high degree of transparency, by publicly broadcasting new transactions and information throughout the network without the necessity of a third party or intermediary [10]. Furthermore, integrity of data [11] is ensured by involving participants in the data verification process and securing interactions via public-key cryptography. In addition, trust is facilitated through the establishment of an immutable architecture [12], which is based upon consensus mechanisms. On the other hand, the decentralized setting facilitates the realization of a private, reliable and versatile environment. A high degree of privacy is reached, as interactions in the peer-to-peer network rely on publickey cryptography, introducing pseudonyms for each participant [13]. Reliability is achieved through a redundant [14] and code-based design with potential for automation [15]. Furthermore, blockchain technology provides a platform, in which every participant is permitted to introduce and distribute their own code and programs and, therefore, offers an open and versatile setting [16].

Ontologies and Ontology Engineering. Ontologies and semantic models are already an established solution for ensuring a common, agreed upon understanding of terms and their relationships within a certain domain. Gruber [17] describes an ontology as "an explicit specification of a conceptualization". In this context, an ontology builds upon a set of concepts with shared and agreed on meaning and a set of formal axioms that constrain the interpretation and well-formed use of these terms [18]. The application areas are manifold. For instance, ontologies deliver the basis for the formal description of complete domains, are used to facilitate data integration, or to automatically deduce new knowledge or recognize fact conflicts.

As blockchain technology is centered around a peer-to-peer network, in which different actors, interfaces, assets, transactions etc., are interconnected and interact with each other, an ontology is well-suited to comprehensively describe inherent elements and components as well as the relationships within blockchain networks. "The goal of such models is to provide a transparent view of the essential elements of the increasingly complex information systems to 
business people in order to better understand the functionality, define requirements and decide about future strategies" [5]. Precisely in this context we develop our ontology for describing blockchain business networks. Following Gruber [17], five distinct design criteria have to be accounted for when developing an ontology:

1. An ontology should be defined as clearly as possible. The goal is to define identified concepts and terms as objective, formalized and completed as possible.

2. The ontology should be coherent, meaning a logically consistent, structure should be aspired.

3. While an ontology provides a formal foundation for the description of concepts, it should be designed in a way that is open for extensions and specialization on the basis of existing definitions and descriptions.

4. Focus should be placed on minimizing the encoding bias. Therefore, representations should be based on a knowledge level.

5. While an ontology is created with a certain knowledge sharing activity in mind, it should follow a minimal ontological commitment, in order to, minimize unintended limitations concerning its descriptive capabilities. Therefore, solely essential terms and underlying theories should be defined.

We follow these criteria and use established ontology engineering approaches [19]-[21] to develop our Blockchain Business network Ontology (BBO), see Section 5.

Model-driven Development. Following a modelbased approach helps to derive an understanding of a system, by bringing together different views with varying levels of abstraction. In this context, a user is enabled to build upon a model that represent a system's details and characteristics, which can be used to fully rebuild the system [22]. Following a model-driven architecture or design contributes to understanding and describing a system in various ways [22]:

1. Since a model builds upon a well-defined notation and typology, the relationships between the distinct elements as well as their descriptions contribute to a general understanding of the system, while also helping to develop scalable solutions.

2. An architectural framework may be used to combine and transform different models and descriptive layers to facilitate the construction of a system.

3. Building upon a set of formalized meta-models, which in turn can be integrated and transformed into models with a higher degree of information, automation may be applied.
4. Industry standards are the essential foundation for increasing both acceptance and adoption for a model-based endeavor.

We build on these fundamentals in order to develop our model-driven approach for describing blockchain business networks.

\section{Related Work}

Blockchain technology has only recently gained on popularity in the context of providing solutions in business ecosystems. Nevertheless, there is already some work on developing ontologies for the technology and on using semantic models for describing the involved business networks. In this section, we focus on reflecting on two main lines of work: 1) ontologies for blockchains and 2) ontologies for describing business networks.

Ontologies for blockchains. Business models are structural templates [23] or architectures [24], which describe the business logic behind the value creation and delivery for a company's customers. In this context, business networks focus on the involved parties, their roles and the way they interact. In this section, we discuss to what extent existing blockchain ontologies can capture business network aspects.

Currently there are two main contributions in the area of semantic modeling for blockchains (see [25], [26]), accompanied by some domain-specific approaches, for instance, in the financial sector. As part of the "Semantic Blockchain" initiative, BLONDiE (Blockchain Ontology with Dynamic Extensibility) [25] aims to provide the basis for describing the blockchain native structure and some related information. For instance, it covers crypto-currencies, transactions, block characteristics, et cetera. It is strongly based on the official specification of Bitcoin and the Ethereum Network, therefore, its concepts reflect mainly the technical functioning of blockchains and not the corresponding participants and interactions within the network. Therefore, BLONDiE is suitable for modeling the technical aspects of transactions, but cannot capture business network characteristics.

The EthOn [26] (Ethereum ontology) aims to provide clear meaning of common blockchain terms and to describe their relations. Similarly, to BLONDiE, the covered concepts are very much determined by the technical specifications of Bitcoin and Ethereum. As a result, EthOn especially enables the description of Ethereum artifacts. Still, neither BLONDiE nor EthOn offer the means for describing business network aspects of blockchain interactions.

As already mentioned, there are also some approaches related to specific industrial sectors, such 
as the financial one. For instance, FIBO (Financial Industry Business Ontology) ${ }^{2}$ is a very rich ontology for the financial services domain. It covers more than 600 classes, of which some have a direct relation to blockchain technology. Hence, it can be aligned, matched or extended with specific blockchain ontologies or individual concepts. In the context of our work, FIBO provides a good example of how blockchains can be modelled in terms of their business or industrial context. Still, the gap of being able to describe the business network aspect within blockchain networks remains unaddressed.

Ontologies for business networks. Ontologies are an established way for describing and analyzing business networks. One of the first initiatives is the REA (Resources Events Agents) ontology, which was originally designed for accounting systems (see [27], [28]) and was later extended with additional concepts in order to support e-commerce and virtual organizations [29]. Similarly, the Business Model Ontology (BMO) [30] targets the need to describe the business model of an enterprise, by considering an enterprise and its environment, in the context of a particular customer's demand. TOVE (Toronto Virtual Enterprise) [31] is another ontology, that aims to capture an enterprise and its properties. It offers an ontology stack, with separate ontologies for enterprise activities, resources, costs, quality, and time. It is not designed to target a particular industry.

Furthermore, there are a number of ontologies that focus on specific aspects of the business networks including trust, product offering and bundling, or the network context and the external business environment. Fatemi et al. [32] provide an ontology for describing trust aspects within a business collaboration setting. Akkermans et al. [33] use an ontology-based analysis regarding bundle offerings for providing a multi-actor business model view for e-service bundles. Targeting the environment of business networks, Lewanska et al. [34] aims to support the process of business environment analysis and to allow for business network identification. The developed ontologies have the goal to capture not only the internal aspects of an organization, but also to be able to describe the external business environment.

Finally, there are also some ontologies developed for describing business aspects for a very specific purpose. For example, the SUPER ontology stack [35], or GoodRelations [36] are developed for modeling ecommerce companies and related products, prices, etc. in order to build semantically annotated e-shops.

\footnotetext{
2 https://www.edmcouncil.org/financialbusiness [Accessed: 15-May-2017]
}

All of the here mentioned ontologies are developed to capture specific parts of the business networks in order to be able to support a better completion of related tasks. Thus, they focus on describing the organization's internal characteristics and relations, or on capturing the communication with external partners. While some of the ontologies provide a good basis for describing general network characteristics, none of them provide the classes and properties that are required for capturing the interactions within a blockchain business network.

\section{Requirements and Methodology}

In this section, we illustrate the requirements and the pursued methodology for developing a modeldriven approach for the description of blockchain business networks. In terms of our methodology, we rely on standard solutions from the areas of ontology engineering and model-driven design (see Section 2).

We define the Blockchain Business Network Ontology (BBO) in three main steps, following the standard for ontology engineering: 1) analysis of a set of use cases in order to determine the level of the ontology, its scope and the main concepts, 2) definition of competency questions in order to determine the needed information coverage, and 3) definition of a set of design principles that guide the ontology design.

Good practices in ontology engineering [17] prescribe a number of criteria that should be met while conceptualizing a new model (see Section 2). We match these to specific design principles (DP) that we aim to follow throughout our work:

DP1. The ontology should cover all necessary common blockchain business network characteristics, focusing on the overall properties, the participating parties and the types of communication (minimizing the encoding bias and minimal ontological commitment).

DP2. The ontology should be extendable to cover different types of communication and communicating parties (extension).

DP3. The ontology should capture the information required for the formalization of an existing network and provide the basis for evaluating (coherent): 1) its suitability for using blockchain technology, and 2) conducting blockchain-oriented network analysis.

DP4. The ontology should be compatible with existing semantic annotation approaches. This should be realizable through ontology matching or conceptual extensions (coherent and clear).

DP5. The ontology should facilitate simplicity of use for creating network descriptions (clear).

DP6. The ontology should aim to be minimal, but capture the necessary information for supporting the 
description of blockchain business networks (coherent and clear).

In this way, we aim to ensure the compatibility, reusability and adaptability of our model-driven approach. We complement these design principles with a set of competency questions (CQ) that specify the information content that needs to be covered by the developed model. These questions were defined by analyzing a set of use cases, which are publicly available at the Use Case Inventory $\mathrm{Wiki}^{3}$.

First, we started by identifying the general blockchain network characteristics, and the information that is needed for the network description. Relevant information in this respect is: (CQ1) "What is the network structure - is it client-server-based or peer-to-peer?", (CQ2) "What is the network architecture - is it centralized, decentralized or distributed?" and finally, (CQ3) "Is the network public, private or requires a case-based permission?". After covering the general network-related characteristics, we move on to the nodes and the edges of the network - the participants and the communication that connects them. (CQ4) "What are the networks participants?" and (CQ5) "Can the same network participant have different roles, depending on the type of communication?". Based on the blockchain specification we know that a participating node can have different roles. For example, in one transaction a node may only be an observer, while in another one, it can be initiating or validating a transaction. This leads us to the question (CQ6) "What are the roles that a participant can have?". Finally, we also consider the type of communication that is covered by asking (CQ7) "How many nodes are involved in a communication?" and (CQ8) "What are possible communication types between nodes?".

The so designed ontology is not bound to any particular annotation formalism. Furthermore, it can be used as an extension to existing network ontologies, by simple creating a matching or extension to the corresponding network and participating element.

In the following section, we introduce our two main contributions - the layer model and the BBO.

\section{Describing Blockchain Business Networks}

In order to support the description of blockchain business networks, we rely on two main building blocks - a layer model (see fig. 1) and an ontology (see fig. 2). The layer model facilitates to capture a comprehensive picture of a blockchain, including business, network and technical aspects, while the ontology complements the layer model by enabling the description of the specific characteristics of a blockchain business network. In this way, we argue that we cover two main dimensions - the vertical one, in terms of a top-to-bottom view on blockchain networks, and the horizontal one, with a detailed description of the network participants and their communication.

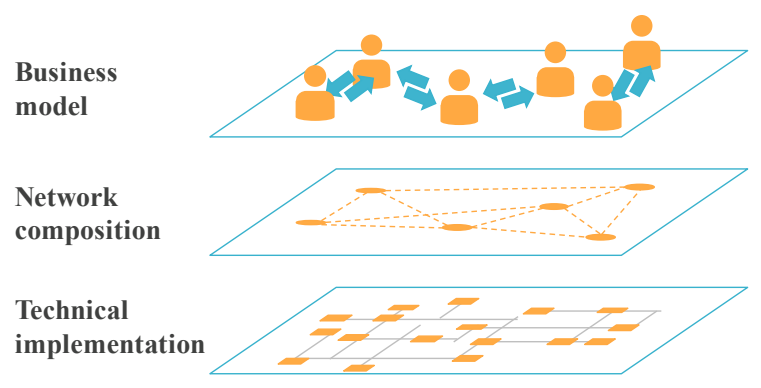

\section{Figure 1 Blockchain layer model}

The blockchain layer model. The development of distinct models, addressing specific and varying aspects of a greater system, helps to gain a comprehensive understanding of that system or phenomenon. Through these models, different levels of abstraction can be assessed, which can afterwards be mapped onto each other or whose content can be propagated to other levels, in order to grasp the entire magnitude of a construct [22]. Therefore, in order to facilitate the description of blockchain-driven business networks in a sound and comprehensive manner, three constitutive layers (see figure 1) are defined. These layers range from a business model view to a codebased perspective. We deem all of these layers as important, since we expect blockchain technology to affect all of them in a novel manner.

The layers are interconnected, such that the technical implementation influences the network composition, which in turn has an impact on the business model. A detailed description as well as differentiation of the layers is provided in the following.

Business model. The highest level of abstraction deals with the description of the business model of a respective business network. Although the notion of the business model is still somewhat ambiguous in academia, it can be seen as a structural template [23] or architecture [24], describing the business logic. In this context, the business model layer provides insights into

\footnotetext{
3 https://wiki.hyperledger.org/groups/requirements/use-caseinventory [Accessed: 20-May-2017]
} 


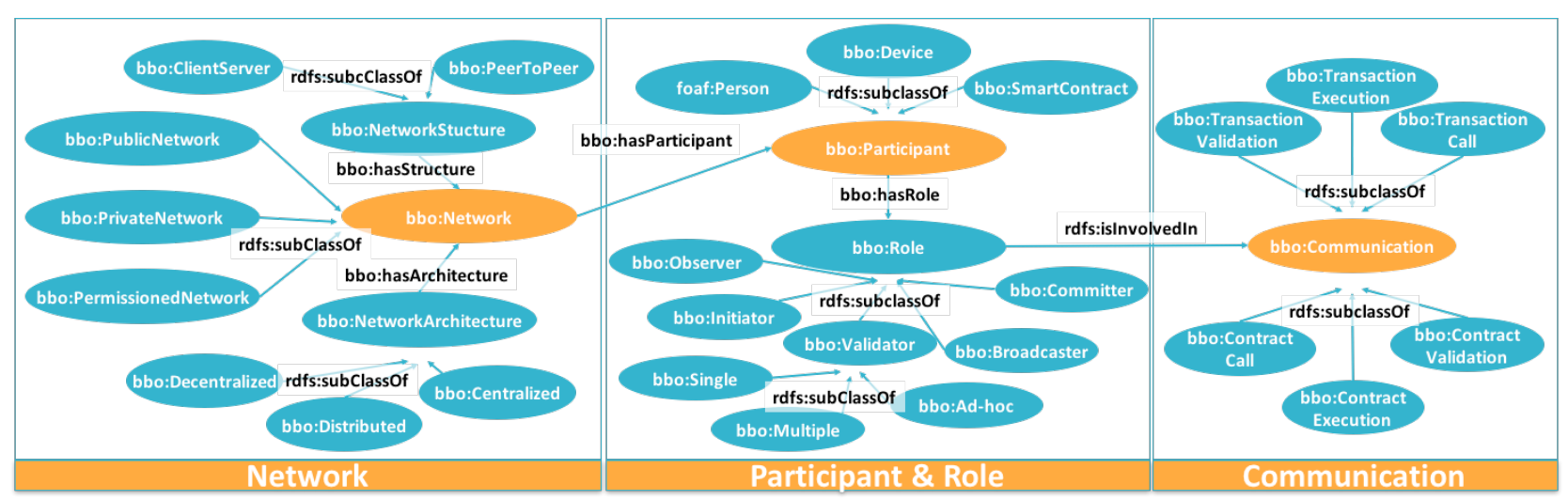

Figure 2 Blockchain Business Network Ontology (BBO)

the different business entities, processes, components and rules in blockchain business networks, while also disclosing their relationships. A starting point for this layer might be the e-Business Model Ontology by Osterwalder and Pigneur [37].

Network composition. Next to taking a business perspective, it is also crucial to understand the basic characteristics, participants and communication patterns of a business network. The Blockchain Business Network Ontology (BBO) mainly focuses on providing the means to describe these aspects. Focus is, therefore, placed on maintaining a platformindependent perspective, which makes it possible to not rely on distinct documentation (such as the existing ones on Ethereum or the Bitcoin network). Therefore, the basic components of a network, namely its characteristics, nodes and edges are depicted.

Technical implementation. The lowest layer of abstraction describes the code-based structure and content of a blockchain business network. In this context, the implementation level describes the general architecture, in which the business network is translated into logical expressions [38].

Based on the presented layers, our research focuses on the description of the network composition layer, since it plays the leading role in deciding whether and how blockchain technologies can be applied in a specific use case. To this end we introduce the BBO.

(BBO) Blockchain Business Network Ontology. In the following we describe the main concepts of the $\mathrm{BBO}$ ontology and, at the same, time check the level of conformity to the competency questions (CQs). Despite the fact that the competency questions were used to determine the necessary information to be covered by the ontology, depending on the way the ontology is realized, complete coverage is not always possible. Therefore, checking CQ conformity is not redundant and in fact necessary.
The $\mathrm{BBO}^{4}$ can be divided up into three main parts, which are illustrated in figure 2 . The first one focuses on the general characteristics of the network, illustrating the respective structure (information coverage (CQ1)), architecture (CQ2) and type of the blockchain business network (CQ3). These characteristics may, for instance, vary depending on the specific blockchain platform, such as Ethereum, Hyperledger etc., or a respective use case. Furthermore, the participants of a network have to be specified, as they are an integral part of the network, representing the nodes in its basic structure (CQ4). A crucial aspect of this part of the ontology is the specification of the distinct roles (CQ5, CQ6), which the participants can have. For instance, we differentiate between an observer, an initiation, a committer, a validator and a broadcaster. These roles can be extended with further classes, should this be necessary. In addition, the different types of network participants are described. These can be further specified by linking to external ontologies, for example to $\mathrm{FOAF}^{5}$, or by using the defined classes for devices and smart contracts. Next to describing the nodes of a network, it is essential to describe their relationships or linkages. In our case, linkages are represented through communication patterns (CQ7, CQ8), which deal with the handling of transactions and contracts.

By following best practices in ontology engineering, we aim to ensure the quality of the developed semantic model. By relying on modeldriven development, we ensure a shared general understanding of the system, since the BBO as an underlying model provides a well-defined notation and typology, distinct elements and their relationships. Furthermore, the combination of the model (e.g. BBO)

\footnotetext{
4 Current version of the BBO [15-June-2017] available at: https://github.com/mmale/Blockchain-Business-Network-Ontology

5 FOAF (friend of a friend) schema: http://www.foaf-project.org [Accessed: 20-May-2017]
} 
and the different descriptive layers can be used as a basis for facilitating the construction of a suitable solution. Finally, a model-driven development approach increases both acceptance and adoption, and at the same time provides grounds for continuous and consistent analysis. An example for this might be the development of approaches for determining the suitability of blockchain solutions for a given network structure or for automatically designing solutions based on the network model.

\section{Evaluation and Use Case Application}

In this section, we evaluate our approach in terms of the conformity to the design principles, as defined in Section 4. We demonstrate the practical applicability of our work via the detailed description and discussion of a use case.

Conformity to design principles and information coverage. Similarly to evaluating the conformity to the competency questions, checking the level of agreement with the design principles might seem superficial. However, depending on the realization of the ontology, sometimes some of the principles may be violated. One commonly violated principle is the minimal ontological commitment, where often, for example, classes that describe different types of the same things are defined, instead of having a property that defines the type. Classes are easier to handle programmatically, so practical implementation is chosen over conformity with the design principles.

In the case of $\mathrm{BBO}$, regarding the design principles, we followed a systematic approach to cover all common blockchain business network characteristics, thus conforming to DP1. We relied on existing network model approaches, as well as on examining a series of use cases. In relation to extendibility, the BBO can, for instance, be modified to include further types of roles and communication patterns. The defined set of classes in not restrictive, therefore, BBO conforms to DP2. Based on the use case described below and relying on several preliminary discussions with fellow researchers, the BBO captures the information required for the formalization of an existing network (DP3), thus providing the basis for analysis and evaluation. Nevertheless, more evaluation needs to be done in terms of how well all relevant properties are captured. Regarding the compatibility with existing semantic annotation approaches (DP4), the BBO does not pose any restrictions or special requirements. Furthermore, it consists of only 3 to 4 main classes, refined via subclasses and types, facilitating the annotation process (DP5). This is also closely connected to DP6, aiming for a minimal but sufficient amount of information that is to be captured. Based on this short discussion, we can summarize that the BBO complies with all design principles, while for some of them a more thorough evaluation might be beneficial. The information coverage in terms of competency questions was already discussed in the previous section, as part of the description of the BBO.

Use case application - Supply chain traceability and anti-counterfeiting. The use case is related to an industry context and was selected from the Use Case Inventory Wiki of the Hyperledger Requirements Working Group (HRWG) ${ }^{6}$, which, among others, documents and describes blockchain use cases, utilizing a pre-defined use case template, while also keeping record of the status and completion of the use case description. Despite the fact, that the selected use case is a theoretical one, which has not been applied in real world, we rely on the use case due to its rich description of its elements and deem the HRWG as a reliable source of information.

Forgery and fake products have a vast impact on today's companies and supply chains. In 2007, the OCED published a study, assessing the effects of product counterfeiting on international trade, in which they concluded that, in 2005 alone, losses due to counterfeiting could have accumulated to as much as USD 200 billion [39]. In this context, a blockchain solution is proposed to automate product tracking and to install condition-based notifications for distinct user groups. In detail, the use case at hand deals with a supply chain, handling potentially counterfeit microchips, which have a distinct digital identity with their own public and private keys, helping to process the product's provenance through the use of singing algorithms. The use case is to be implemented in a permissioned and decentralized blockchain, where accessing the network is limited through a membership service. The following five types of supply chain participants are introduced: Customs, broker, seller, buyer, OEM and endorser. All participants in the regarded supply chain have a role in enabling traceability. So, apart from being involved in a trading relationship, all participants are responsible for ensuring traceability of the product. The tracking process starts with a customs inspection.

In a permissioned setting the question arises if the access to data should be managed publicly or privately. In case of the supply chain inquiry, the HRWG suggests that data should solely be shared in a direct manner with neighboring trading partners, which are one tier up- or downstream the respective company.

\footnotetext{
6 https://wiki.hyperledger.org/groups/requirements/requirements-wg [Accessed: 20-May-2017]
} 


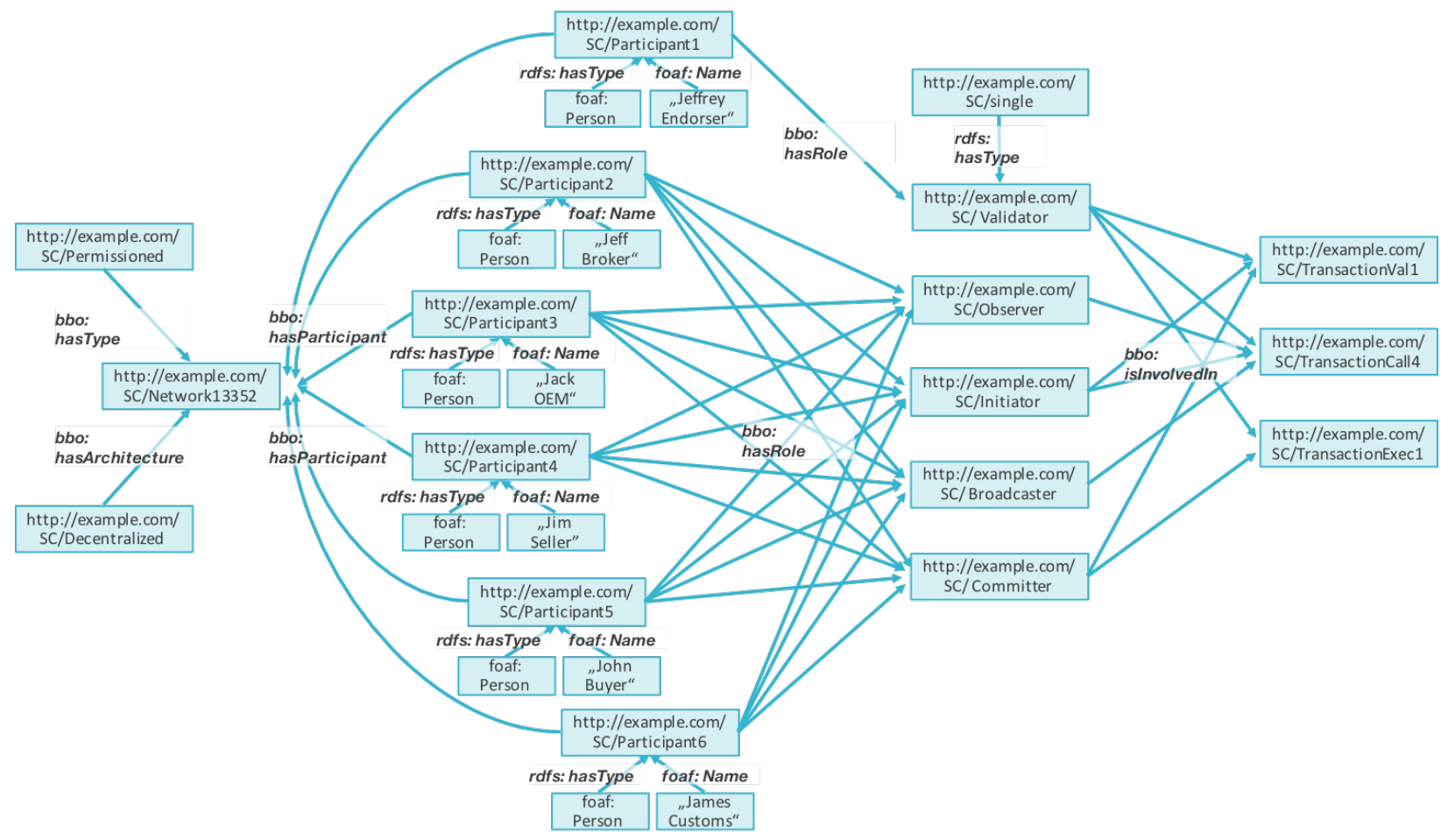

Figure 3 Supply chain traceability and anti-counterfeiting use case modelled with BBO

In this context, so-called endorsers fulfill the role of validating the data. Therefore, in case a supply chain participant receives a microchip, wants to examine and provide proof of the authenticity of the product, the participant or client has to approach the endorsing party to validate the associated data. The endorser then checks the underlying chaincode and confirms a specific transaction before it is committed by the client, which in our case might be an update of the Digital Identity of the product, labeling it as a counterfeit product.

Based on the provided use case an instantiation of the developed $\mathrm{BBO}$ is conducted and is illustrated in figure 3. In this example, a decentralized and permissioned blockchain network is modeled. A set of entities with various roles are integrated into the network, while also illustrating their communication activities (cf. table 1).

Evaluating the instantiation discloses that the BBO is suitable for describing the integral parts of the blockchain network. In this context, the ontology provides the means to model the various relationships between the different entities and components, which can be found on a network composition level. Looking at the presented use case, the ontology offers a solution to render the case description machine readable.

Based on the prose text, we were able to extract the different entities, their rolls as well as their communication and match it to our ontology. Next to reading, understanding and displaying the relationships of the use case description, it is now even possible perform analysis and automation, e.g. concerning the creation of such a network.

\section{Table 1. Use case elements}

\begin{tabular}{|c|c|c|}
\hline Entity & Rolls & Communication \\
\hline $\begin{array}{l}\text { Customs, } \\
\text { Broker, } \\
\text { Seller, } \\
\text { Buyer, } \\
\text { OEM }\end{array}$ & $\begin{array}{l}\text { Observer } \\
\text { Initiator } \\
\text { Broadcaster } \\
\text { Committer }\end{array}$ & $\begin{array}{l}\text { Initializes transactions to } \\
\text { confirm location and status } \\
\text { of product; executes } \\
\text { verified transactions; Calls } \\
\text { current status of the } \\
\text { product }\end{array}$ \\
\hline End & Validator & $\begin{array}{l}\text { Calls previous status of the } \\
\text { product; } \\
\text { transactions }\end{array}$ \\
\hline
\end{tabular}

\section{Conclusion}

In order to drive research efforts and the overall adoption of blockchain technology, we contribute to the establishment of a common understanding of the interactions and structure in blockchain business networks. First, we provide a layer-based model, presenting constitutive levels of abstraction for the comprehensive description of blockchain business 
networks. As these layers range from taking a business model view to a technical implementation perspective, a sound picture of the various aspects of a blockchain business network is created. Second, the Blockchain Business Network Ontology (BBO) is introduced, providing the means to depict the integral parts of a blockchain network. In this context, the ontology serves as basis for the analysis of existing and development of aspired blockchain business networks. Third, we show the practical applicability of our approach by assessing a publicly available blockchain use case of the Hyperledger Requirements Working Group (HRWG).

As part of future work, we plan on conducting a coverage evaluation of our approach, by taking use cases, with different characteristics into consideration. For instance, although we deem the use case description of the HRWG as information-rich, we heavily rely on its correctness. Since the selected case has not yet been applied to reality, we need to take additional use cases into account. Therefore, cases originating from different blockchain platforms, such as Ethereum or Ripple, with varying structural compositions and consensus mechanisms need to be taken into account. This is especially challenging, as, until now, there is a lack of rich use case descriptions meeting these requirements.

Furthermore, we strive to perform a large-scale analysis of existing blockchain use cases, collecting data by applying the developed ontology. Thereby, a structured and systematic assessment of blockchain applications is facilitated, opening up the opportunity to discover typical patterns in blockchain-driven networks. Thereby extending the body of knowledge concerning blockchain on a business network level.

\section{References}

[1] D. Tapscott and A. Tapscott, Blockchain Revolution: : How the Technology Behind Bitcoin Is Changing Money, Business, and the World. New York: Penguine Random House, 2016.

[2] J. Amaral, E. G. J. Anderson, and G. G. Parker, "Putting It Together: How to Succeed in Distributed Product Development," MIT Sloan Manag. Rev., vol. 52, no. 2, pp. 51-58, 2011.

[3] Y. Lewenberg, Y. Sompolinsky, and A. Zohar, "Inclusive Block Chain Protocols," in FC 2015, vol. 1, R. Böhme and T. Okamoto, Eds. Berlin, Heidelberg: Springer Berlin Heidelberg, 2015, pp. 528-547.

[4] B. Warburg, "How the blockchain will radically transform the economy," 2017. [Online]. Available: goo.g1/19MnpJ. [Accessed: 21-Nov-2016].
[5] A. Albani and J. L. G. Dietz, "Enterprise ontology based development of information systems," Int. J. Internet Enterp. Manag., vol. 7, no. 1, pp. 41-63, 2011.

[6] S. Nakamoto, "Bitcoin: A Peer-to-Peer Electronic Cash System," 2008.

[7] J. Garay, A. Kiayias, and N. Leonardos, "The Bitcoin backbone protocol: Analysis and applications," in Lecture Notes in Computer Science, vol. 9057, E. Oswald and M. Fischlin, Eds. Berlin, Heidelberg: Springer Berlin Heidelberg, 2015, pp. 281-310.

[8] F. Tschorsch and B. Scheuermann, "Bitcoin and Beyond: A Technical Survey on Decentralized Digital Currencies," IEEE Commun. Surv. Tutorials, vol. 18, no. 3, pp. 2084-2123, 2016.

[9] S. Seebacher and R. Schüritz, "Blockchain Technology as an Enabler of Service Systems: A Structured Literature Review," in Exploring Services Science: 8th International Conference, IESS 2017, Rome, Italy, May 24-26, 2017, Proceedings, S. Za, M. Druagoicea, and M. Cavallari, Eds. Cham: Springer International Publishing, 2017, pp. 12-23.

[10] R. Beck, J. Stenum Czepluch, N. N. Lollike, and S. Malone, "Blockchain - The Gateway to trust-free cryptographic Transactions," in 24th European Conference on Information Systems (ECIS), 2016, pp. $1-14$.

[11] K. Delmolino, M. Arnett, A. E. Kosba, A. Miller, and E. Shi, "Step by Step Towards Creating a Safe Smart Contract: Lessons and Insights from a Cryptocurrency Lab," IACR Cryptol. ePrint Arch., vol. 2015, p. 460, 2015.

[12] J. Cucurull and J. Puiggalí, "Distributed immutabilization of secure logs," Lect. Notes Comput. Sci., vol. 9871, pp. 122-137, 2016.

[13] R. Hull, V. S. Batra, Y. M. Chen, A. Deutsch, F. F. T. Heath, and V. Vianu, "Towards a shared ledger business collaboration language based on data-aware processes," in Lecture Notes in Computer Science, vol. 9936 LNCS, Q. Z. Sheng, E. Stroulia, S. Tata, and S. Bhiri, Eds. Cham: Springer International Publishing, 2016, pp. 18-36.

[14] M. Sharples and J. Domingue, "The blockchain and kudos: A distributed system for educational record, reputation and reward," in Lecture Notes in Computer Science, vol. 9891 LNCS, K. Verbert, M. Sharples, and T. Klobučar, Eds. Cham: Springer International Publishing, 2016, pp. 490-496.

[15] I. Weber, X. Xu, R. Riveret, G. Governatori, A. Ponomarev, and J. Mendling, "Untrusted business process monitoring and execution using blockchain," Lect. Notes Comput. Sci., vol. 9850, pp. 329-347, 2016. 
[16] S. Ølnes, "Beyond Bitcoin enabling smart government using blockchain technology," Lect. Notes Comput. Sci., vol. 9820, pp. 253-264, 2016.

[17] T. R. Gruber, "Toward principles for the design of ontologies used for knowledge sharing?," Int. J. Hum. Comput. Stud., vol. 43, no. 5, pp. 907-928, 1995.

[18] J. Cardoso and A. Sheth, "Introduction to Semantic Web Services and Web Process Composition," in SWSWPC 2004, J. Cardoso and A. Sheth, Eds. Berlin, Heidelberg: Springer Berlin Heidelberg, 2005, pp. 113.

[19] P. Hitzler, A. Gangemi, and K. Janowicz, Ontology Engineering with Ontology Design Patterns., vol. 25. IOS Press, 2016.

[20] A. Gomez-Perez, O. Corcho-Garcia, and M. Fernandez-Lopez, Ontological engineering. Springer/Heidelberg, 2003.

[21] N. F. Noy and D. L. McGuinness, "Ontology Development 101: A Guide to Creating Your First Ontology," 2001.

[22] A. W. Brown, J. Conallen, and D. Tropeano, "Introduction: Models, Modeling, and Model-Driven Architecture (MDA)," in Model-Driven Software Development, S. Beydeda, M. Book, and V. Gruhn, Eds. Berlin, Heidelberg: Springer Berlin Heidelberg, 2005, pp. 1-16.

[23] R. Amit and C. Zott, "Value creation in E-business," Strateg. Manag. J., vol. 22, no. 6-7, pp. 493-520, Jun. 2001.

[24] D. J. Teece, "Business Models, Business Strategy and Innovation," Long Range Plann., vol. 43, no. 2-3, pp. 172-194, Apr. 2010.

[25] H. Ugarte, "A more pragmatic Web 3.0: Linked Blockchain Data," https://semanticblocks.wordpress.com, 2017. [Accessed: 21-April-2017].

[26] J. Pfeffer, "EthOn - An Ethereum Ontology." http://ethon.consensys.net, 2017. [Accessed: 21-April2017].

[27] G. L. Geerts and W. E. McCarthy, "An accounting object infrastructure for knowledge-based enterprise models," IEEE Intell. Syst., pp. 1-6, 1999.
[28] W. E. McCarthy, "The REA Accounting Model - A Generalized Framework for Accounting Systems in a Shared Data Environment," The Accounting Review, vol. 57, no. 3. pp. 554-578, 1982.

[29] G. Geerts and W. McCarthy, "An ontological analysis of the economic primitives of the extended - REA enterprise information architecture," Int. J. Account. Inf. Syst, vol. 3, no. 1-18. 2002.

[30] A. Osterwalder, "The Business Model Ontology - A Proposition in a Design Science Approach,", 2004.

[31] M. S. Fox, M. Barbuceanu, M. Gruninger, and J. Lin, " $\{\mathrm{A}\} \mathrm{n}\{\mathrm{O}\}$ rganisation $\{\mathrm{O}\}$ ntology for $\{\mathrm{E}\}$ nterprise $\{\mathrm{M}$ \}odeling," Simulating Organ. Comput. Model. Institutions Groups, no. 7, pp. 131-152, 1998.

[32] H. Fatemi, M. van Sinderen, and R. Wieringa, "A Trust Ontology for Business Collaborations.," in PoEM (Short Papers), 2012, vol. 933.

[33] H. Akkermans, Z. Baida, J. Gordijn, A. Z. Morch, and H. Saele, "Ontology-Based Analysis Of eService Bundles For Networked Enterprises.," in BLED 2004 Proceedings, 2004, p. 31.

[34] E. Lewanska and M. Kaczmarek, "Ontologies for Business Networks Identification," in FOMI, 2015, vol. 225, pp. 13-24.

[35] A. Filipowska, M. Kaczmarek, M. Kowalkiewicz, I. Markovic, and X. Zhou, "Organizational ontologies to support semantic business process management," in Proceedings of the 4th International Workshop on Semantic Business Process Management. ACM, 2009, pp. 35-42.

[36] M. Hepp, “\{GoodRelations $\}$ : An Ontology for Describing Products and Services Offers on the Web," in $E K A W, 2008$, vol. 5268, pp. 329-346.

[37] A. Osterwalder and Y. Pigneur, "An e-business model ontology for modeling e-business," 15th Bled Electron. Commer. Conf. June 17-19, p. 12, 2002.

[38] S. J. Russell and P. Norvig, Artificial Intelligence: A Modern Approach, 1st ed. Upper Saddle River, NJ, USA: Prentice-Hall, Inc., 1995.

[39] OECD, "The Economic Impact of Counterfeiting and Piracy." 2007. 